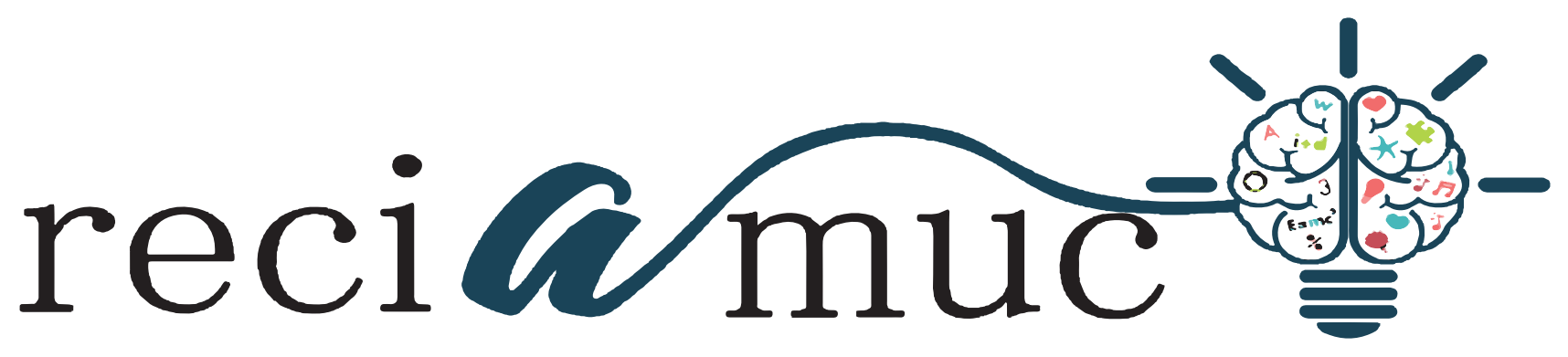

DOI: 10.26820/reciamuc/5.(4).noviembre.2021.37-47

URL: https://reciamuc.com/index.php/RECIAMUC/article/view/718

EDITORIAL: Saberes del Conocimiento

REVISTA: RECIAMUC

ISSN: 2588-0748

TIPO DE INVESTIGACIÓN: Artículo de Revisión

CÓDIGO UNESCO: 5605 Legislación y Leyes Nacionales

PAGINAS: $37-47$

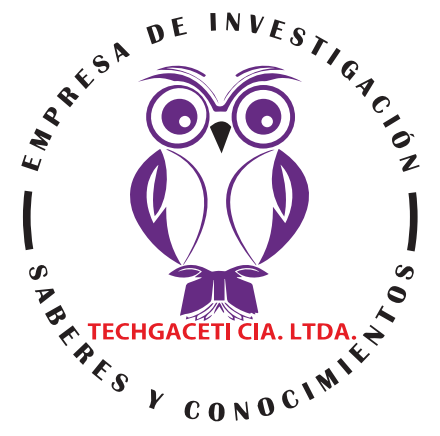

\title{
Estrategias de políticas públicas de salud sexual y reproductiva. Despenalización del aborto en Ecuador
}

Public policy strategies for sexual and reproductive health. Decriminalization of abortion in Ecuador

Estratégias de políticas públicas para saúde sexual e reprodutiva. Descriminalização do aborto no Equador

\section{Sabina Lorena Gamboa Vargas'; Efrén Ernesto Guerrero Salgado²}

\section{RECIBIDO: 15/09/2021 ACEPTADO: 05/10/2021 PUBLICADO: 29/11/2021}

1. Master Universitario en Protección Internacional de los Derechos Humanos; Abogada de los Juzgados y Tribunales de la Republica del Ecuador; ; Maestría en Derecho con mención en Gestión Pública Pontificia Universidad Católica del Ecuador sede Ambato; Universidad Tecnológica Indoamérica; sabinagamboa@uti. edu.ec; (iD https://orcid.org/0000-0003-2591-4071

2. Doctor en Gobierno y Administración Pública, Universidad Complutense de Madrid; Pontificia Universidad Católica del Ecuador; Quito, Ecuador; eeguerrero@puce.edu.ec; http://orcid.org/0000-0003-4636-0362

\section{CORRESPONDENCIA \\ Sabina Lorena Gamboa Vargas \\ sabinagamboa@uti.edu.ec}

Ambato, Ecuador

() RECIAMUC; Editorial Saberes del Conocimiento, 2021 


\section{RESUMEN}

Según cifras de la Organización de Naciones Unidas (ONU, 2019) cada día siete menores de edad se convierten en madres en Ecuador. Por su parte, el Ministerio de Salud Pública estima que el 15.6\% de muertes maternas se deben a abortos realizados en la clandestinidad. Este comportamiento afecta el desarrollo integral de niñas y adolescentes, pues se vulneran Derechos Humanos como el derecho a la salud, el acceso a la educación o el derecho a una vida libre de violencia. El presente artículo pretende analizar los resultados de la implementación en Ecuador de la Estrategia Nacional Intersectorial de Prevención del Embarazo y Planificación Familiar - ENIPLA y el Plan Familia, dos políticas públicas implementadas bajo el gobierno del ex presidente Rafael Correa. La metodología que se propone es descriptiva, lo que, permite analizar los resultados de la aplicación de políticas públicas de salud sexual y reproductiva en Ecuador.

Palabras clave: Políticas públicas; derechos sexuales y reproductivos; aborto clandestino.

\section{ABSTRACT}

According to data from the United Nations (UN, 2019), every day, seven children become mothers in Ecuador. Additionally, the Ecuadorian Ministry of Public Health says that $15.6 \%$ of maternal deaths happen because of clandestine abortions. This conduct affects the integral development of girls and teenagers due to the fact that Human Rights such as the right to health, access to education and the right to a free-of-violence life are violated. The present article is aimed to analyze the results obtained during the execution of the "Estrategia Nacional Intersectorial de Prevención del Embarazo y Planificación Familiar - ENIPLA" (National Intersectoral Strategy for Pregnancy Prevention and Family Planning) and "Plan Familia" (Family Plan), which were two public policies implemented during former President Rafael Correa government. The study has a descriptive methodology, which makes it to possible to analyze the results of applying public policies on sexual and reproductive health in Ecuador.

Keywords: Public Policies, sexual and reproductive rights, clandestine abortion.

\section{RESUMO}

Segundo dados da Organização das Nações Unidas (ONU, 2019), a cada dia sete crianças tornam-se mães no Equador. Além disso, o Ministério de Saúde Pública do Equador afirma que 15,6\% das mortes maternas acontecem por causa de abortos clandestinos. Essa conduta afeta o desenvolvimento integral de meninas e adolescentes pelo fato de serem violados Direitos Humanos como o direito à saúde, o acesso à educação e o direito a uma vida sem violência. O presente artigo tem como objetivo analisar os resultados obtidos durante a execução da "Estrategia Nacional Intersetorial de Prevención del Embarazo y Planificación Familiar - ENIPLA" (Estratégia Intersetorial Nacional para a Prevenção da Gravidez e Planejamento Familiar) e "Plano Família" (Plano Familiar), que foram duas políticas públicas implementadas durante o governo do ex-presidente Rafael Correa. O estudo tem metodologia descritiva, o que permite analisar os resultados da aplicação de políticas públicas de saúde sexual e reprodutiva no Equador.

Palavras-chave: Políticas públicas, direitos sexuais e reprodutivos, aborto clandestino. 


\section{Introducción}

La salud sexual y reproductiva (SSR) se torna en un eje fundamental para el desarrollo personal del ser humano, pero los factores culturales, sociales, políticos y económicos en el desarrollo de las oportunidades de acceso a esta información revelan la existencia de una brecha palpable en las distintas agrupaciones sociales.

En 1946 la Organización Mundial de la Salud (OMS) definió a la salud como: "un estado de completo bienestar físico, mental y social, y no solamente la ausencia de afecciones o enfermedades. El goce del grado máximo de salud que se pueda lograr es uno de los derechos fundamentales de todo ser humano sin distinción de raza, religión, ideología política o condición económica o social" (OMS, 1946). Con esta definición la premisa es que se concibe a la salud no únicamente como la ausencia de dolencia, si no, mantiene una visión de carácter mental y social que alude bienestar y al goce del estado de salud en todos los ámbitos de nuestra vida, asociándose a la satisfacción personal. Se convierte en un derecho fundamental, lo que obliga a los estados a garantizar el acceso en todas sus circunstancias.

Por otra parte, la Carta de Ottawa para la Promoción de Salud de 1986 aporta diferentes enfoques al concepto de salud pública y colectiva definido a esta como "el estado integrador del bienestar humano individual, social y económico, y la promoción de la salud como la vía para la reducción de las desigualdades y el desarrollo del máximo potencial de salud, siendo necesarias condiciones previas favorables como educación, vivienda, alimentación, ingresos, ecosistemas estables, acción comunitaria, justicia social y paz mundial" (Rodríguez, 2016, p.19).

Para Rodríguez (2016) la Declaración de Yakarta sobre promoción de salud hacia el siglo XXI, al igual que la declaración de Alta Mar, constituyeron referentes mundiales al reivindicar la salud como un derecho humano necesario para el desarrollo tanto social como económico.

Por su parte, la Conferencia Internacional de Población y Desarrollo (CIPD) realizada en la ciudad de El Cairo en 1994 estableció las estrategias para mejorar la situación de las niñas y adolescentes, el estatus de la mujer frente al acceso a la SSR, a la igualdad y perspectiva de género, como ejes fundamentales para el desarrollo económico, político y social de los grupos más afectados. La Conferencia Internacional de Población y Desarrollo es una de las convergencias internacionales más importantes en cuestión SSR. Posterior a la asamblea, se crea un Plan de Acción de la CIPD que está dividido en 16 capítulos y aborda temas fundamentales, entre ellos la Igualdad y equidad entre los sexos y habilitación de la mujer, Derechos y salud reproductivos, Salud, morbilidad y mortalidad entre otros fundamentales (Programa de Acción de la CIPD, 1994).

En Ecuador, la salud es reconocida como uno de los deberes primordiales, así, el numeral 1 del artículo 3 establece que es deber del Estado "garantizar sin discriminación alguna el efectivo goce de los derechos establecidos en la Constitución y en los instrumentos internacionales, en particular la educación, la salud, la alimentación, la seguridad social y el agua para sus habitantes" (CRE, 2008).

Es el estado ecuatoriano el que articulará políticas públicas encaminadas al desarrollo progresivo de los derechos conforme lo establece el numeral 8 del artículo 11 de la Constitución, que determina que "el contenido de los derechos se desarrollará de manera progresiva a través de las normas, la jurisprudencia y las políticas públicas. El Estado generará y garantizará las condiciones necesarias para su pleno reconocimiento y ejercicio" (CRE, 2008).

En este sentido, las políticas publicas son definidas por Salazar (2009) como el con- 
junto de sucesivas respuestas del Estado frente a las situaciones consideradas socialmente como problemáticas. La Constitución de la República del Ecuador (CRE) en su Artículo 280 establece que:

Plan Nacional de Desarrollo es el instrumento al que se sujetarán las políticas, programas y proyectos públicos; la programación y ejecución del presupuesto del Estado; y la inversión y la asignación de los recursos públicos; y coordinar las competencias exclusivas entre el Estado central y los gobiernos autónomos descentralizados. Su observancia será de carácter obligatorio para el sector público e indicativo para los demás sectores. (CRE, 2008)

Las políticas públicas en materia de Derechos Sexuales y Reproductivos (DDSSRR) en Ecuador, han tenido un amplio recorrido. El establecimiento de planes a larga data, son los que marcan las pautas de planes sectoriales. El título VI de Régimen de Desarrollo de la Constitución de Ecuador marca la planificación para garantizar el ejercicio de los derechos, por ello, el Plan Nacional de Buen Vivir (2009-2013) fue el instrumento del Gobierno Nacional del ex presidente Rafael Correa para articular políticas públicas. Este plan contaba con 12 Estrategias Nacionales y 12 Objetivos Nacionales que tenían la finalidad de afianzar en Buen Vivir.

Una de las estrategias planteadas fue la Estrategia Nacional Intersectorial de Prevención del Embarazo y Planificación Familiar - ENIPLA (2012-2014). Esta estrategia intersectorial surge como propuesta para apalear las inequidades sociales que se reflejaban en el aceso a SSR. De acuerdo al diagnóstico realizado, en Ecuador los derechos sexuales y derechos reproductivos se encontraban afectados por inequidades económicas, de género y sociales, y fruto de estas inequidades se observaba una tasa diferenciadas de fecundidad en los distitos grupos poblacionales, en la tasa de embarazos no deseados o en el porcentaje de fecundidad precoz existente en la pobla- ción (Ministerio Coordinador de Desarrollo Social, 2011).

A pesar de que la ENIPLA fue ampliamente trabajada desde el ya desaparecido Consejo Nacional de las Mujeres y junto a organizaciones de mujeres jóvenes como la Coordinadora Política Juvenil y, apuntaba a un cambio de enfoque en donde se prime el conocimiento sobre los DDSSR (Guardéras \& Carófilis, 2020), esta estrategia fue reemplazada por el Plan Familia.

Bajo decreto 491 del 26 de noviembre de 2014, la Presidencia de la República, asumió la rectoría del Plan Nacional de Fortalecimiento de la Familia. Este plan tenía como objetivo "prevenir el embarazo adolescente, fortaleciendo el rol protagónico de la familia y empoderando a los y las adolescentes de manera que puedan tomar decisiones libres, informadas y responsables" (Presidencia de la República del Ecuador, 2015, p.33), generando un quiebre con políticas progresistas en materia de DDSSRR. Para Rodríguez (2019) "el abordaje de este cambio de políticas públicas demostró que las decisiones políticas que se llevan a cabo en Ecuador se encuentran mediadas por las representaciones generadas a partir de los medios de comunicación, incluyendo aquellos denominados públicos" (p.3).

\section{2.- Metodología}

La metodología que se propone es descriptiva, lo que, permite analizar los objetivos y resultados de la aplicación de políticas públicas de salud sexual y reproductiva en Ecuador, en particular la ENIPLA y el Plan Familia y compararlas con políticas públicas similares de otros países.

A partir de la descripción se pretende determinar las acciones y programas implementados referentes a salud sexual y reproductiva en Ecuador durante el mandato del ex presidente Rafael Correa.

Para ello se realizará una revisión bibliográfica referente a políticas públicas en materia 
de DDSSRR, así como también una revisión de normativa internacional vigente y de recomendaciones realizadas por diferentes organismos internacionales de protección de derechos.

Se comparará los objetivos planteados es las dos políticas públicas y los resultados de las mismas. Las fuentes utilizadas será secundarias, pues se recurrirá a documentos y datos obtenidos por las instituciones gubernamentales.

\section{3.- Desarrollo}

Ante la necesidad de abordar el índice de fecundidad y embarazos en adolescentes en la región Andina, se consolida gracias al Fondo de Población de Naciones Unidas el Plan Andino de prevención de embarazos en adolescentes (Paz, 2018), este plan reconocía que el embarazo no planificado en adolescentes de la subregión es un problema de Salud Pública, por cuanto era necesario declarar como prioritario los planes de prevención y atención integral (Ministerio de Inclusión Económica y Social, 2010).

De acuerdo con los datos, se estimaba que en Ecuador 2 de cada 3 adolescentes de 15 a 19 años sin educación son madres o están embarazadas por primera vez. La tendencia del incremento del embarazo en adolescentes menores de 15 años en la última década, es del $74 \%$ y en mayores de 15 años es del 9\%. La tendencia de fecundidad adolescente en Ecuador es la más alta de la región andina, llegando a 100×1000 NV (Plan Andino para la Prevención del Embarazo en Adolescentes 2008).

Ante esta realidad el tema de SSR marcó el discurso en la palestra pública por lo que se propuso un trabajo intersectorial coordinado a través de los Ministerios de Educación, Salud, Inclusión Económica y Social, Coordinador de Desarrollo Social, así mismo se coordinó con el Consejo Nacional de Mujeres (CONAMU), Consejo Nacional de la Niñez y Adolescencia (CNNA), Proyecto Ecuador Adolescente de la mano de las ONG's Plan Ecuador y UNFPA, de este trabajo se presentó el Plan Nacional de Prevención del Embarazo en Adolescentes en Ecuador (Paz, 2018).

A pesar de que el Plan Nacional no contó con el apoyo político para su ejecución, los avances en el reconocimiento de la salud sexual y reproductiva como un derecho se gestarían en lo posterior en la Asamblea Constituyente del 2008 (Paz, 2018). Este plan a su vez da un punto de partida para la elaboración de una estrategia intersectorial que marcó un antes y un después en la política pública de SSR.

En Ecuador, según el Ministerio de Inclusión Económica y Social la Tasa Global de Fecundidad (TGF) pasó de 6.7 alrededor de 1950, a 2.4 al 2011. Pese a la disminución existe grupos poblacionales con una transición mas tardía, según el Censo 2010, la TGF es de 2.4. Por otro lado, mujeres no pobres por necesidades básica insatisfechas (NBI) tienen una TGF de 1.9, es decir 0.5 hijos menos en promedio que el nivel nacional, mientras que las mujeres pobres por $\mathrm{NBI}$ Extremo, tienen 3.5 hijos/as por mujer, un hijo adicional sobre el índice nacional, y 1.6 hijos más que las mujeres no pobres, por su parte, la TGF de mujeres indígenas es de 3.3, un hijo adicional sobre la media nacional y las mujeres afroecuatorianas y montubias tienen en promedio 2.8 y 2.7 hijos/as respectivamente (Ministerio Coordinador de Desarrollo Social, 2011).

Es importante señalar que el nivel de educación es un factor que incide en la TGF, pues se constata que "aquellas con ningún nivel de instrucción formal y mujeres con educación hasta primaria, tienen 2.9 y 3.2 hijos respectivamente, mientras que las mujeres con nivel de instrucción superior o más, tienen 1.7. Es decir, existe una asociación negativa entre fecundidad y educación. A medida que las mujeres tienen mejores oportunidades educativas, disminuyen su fecundidad" (Ministerio Coordinador de Desarrollo Social, 2011, p.6) 
En ese sentido, Rozada (2009) ( citado por Ministerio Coordinador de Desarrollo Social, 2011) estima que el grupo poblacional que mayormente se ve afectado con embarazos adolescentes son aquellas con niveles socioeconómicos bajos, debido a múltiples factores como nula educación formal, educación primaria incompleta y bajos conocimientos de SSR.

Por otra parte, entre 1998 y 2009 se registraron 225 mil egresos hospitalarios por aborto en los servicios del Ministerio de Salud. En aproximadamente una década se incrementó de once mil abortos a veinte y cinco mil, es decir más del doble. Estas cifras posicionaban al Ecuador como el país con más abortos en condiciones de riesgo en América Latina (Ministerio Coordinador de Desarrollo Social, 2011).

Abordado el tema de Planificación Familiar, el nivel de conocimiento de al menos un método anticonceptivo varía del 97.8 por ciento entre la población mestiza al 74.9 por ciento entre la indígena. De igual manera se puedo concluir que a mayor nivel de instrucción y nivel económico de las mujeres, es mayor la proporción que tiene conocimiento de los métodos (Centro de Estudios de Población y Desarrollo Social, 2005)

Bajo este panorama, durante el segundo mandato del entonces presidente del Ecuador, el economista Rafael Correa se presentó la ENIPLA. La Estrategia Nacional Intersectorial de Prevención del Embarazo y Planificación Familia que fue diseñada en el 2011 tenía como fin "mejorar la calidad de vida de las ciudadanas y los ciudadanos del país garantizando el ejercicio de los derechos y la salud sexual y reproductiva especialmente de los grupos de atención prioritaria" (Ministerio Coordinador de Desarrollo Social, 2011, p.38). Esta estrategia estaría a cargo en un inicio del Ministerio de Salud Pública para después compartir competencia con los Ministerios de Educación e Inclusión Económica y Social bajo la coordinación del Ministerio Coordinador de Desarrollo Social.
En ese contexto, la metodología planteaba un trabajo intersectorial y participativo, en donde la sociedad civil tenía voz activa en la construcción e implementación de la estrategia. Durante el enlace ciudadano No. 209, el ex presidente destacó lo necesario que era establecer un control de la natalidad y que para ello era indispensable recurrir al uso de anticonceptivos naturales y artificiales, sin embargo, fue enfático en recalcar su postura apegada a los valores católicos y a la doctrina de la iglesia, planteando una fuerte postura en contra de métodos abortivos.

Según datos del Ministerio Coordinador de Desarrollo Social el monto al que ascendía el proyecto era de \$32.610.421,00 (TREINTA Y DOS MILLONES SEISCIENTOS DIEZ MIL CUATROCIENTOS VEINTE Y UNO/100 Dólares de los Estados Unidos de América) y con una cobertura a nivel nacional.

La ENIPLA contó con 4 componentes par alcanzar el objetivo propuesto. En primer lugar, la estrategia le apostaba a la educación sexual basada en un conocimiento científico y con enfoque de derechos para empoderar a los jóvenes en la toma de decisiones. Este componente propició el diseñó e implementación de estrategias metodológicas y subsistemas de información locales, así como fortalecer las líneas telefónicas directas de información.

Bajo la perspectiva del componente dos, se planificó la producción y reproducción de campañas educomunicacionales con el fin de cambiar patrones socioculturales sobre la sexualidad y las relaciones inequitativas entre géneros.

Los servicios de SSR también fueron considerados en la estrategia, para lo que se propuso la adecuación de centros de atención en todo el territorio nacional.

Uno de los programas ampliamente difundidos tanto en medios masivos televisados así como también en escuelas y colegios fue la campaña "Habla serio, sexualidad 
sin misterios" que buscaba promover el diálogo sobre sexualidad, como una manera efectiva de garantizar los derechos de las personas a la información, la salud sexual y reproductiva y la participación (Habla Serio, Sexualidad Sin Misterios | La Iniciativa de Comunicación, n.d.).

Burneo et al. (2015), citado por Cifuentes (2016) menciona que "para muchos investigadores y activistas vinculados a la temática, ENIPLA representó aquello por lo cual muchos colectivos habían luchado. Los feminismos históricos en Ecuador, colectivos feministas, asociaciones que trabajan en torno a la diversidad y los derechos sexuales, organizaciones no gubernamentales de salud pública, salud sexual y reproductiva, activistas involucrados con los derechos de las mujeres, empujaron proceso de adquisición de derechos" (p.78).

Esta estrategia constituyó un puntal de tensión entre posturas progresistas y conservadoras, es así que en Ecuador empezaron a surgir colectivos ciudadanos que estaban en contra de la implementación de esta estrategia que promovía el uso de anticonceptivos en adolescentes. Varios actores políticos y religiosos se pronunciaron en contra, es así que el ex mandatario en el 2015 desestimó la estrategia, la acusó de extralimitarse y la consideró una política que promovía el hedonismo en los adolescentes (Yépez, 2021).

Las presiones de ciertos grupos conservadores allegados al Opus Dei lograron que a través del decreto Ejecutivo No. 941 el ex Presidente reemplazara en el 2014 la ENIPLA por el Plan Familia que estaría dirigido por la Dra. Mónica Hernández. Este reemplazo constituyó un retroceso en los derechos adquiridos en materia de SSR.

A diferencia de la ENIPLA que fue cordinada de manera intersectorial, el plan familia estuvo a cargo de la Presidencia de la República del Ecuador, el Ministerio de Educación, Ministerio de Salud Pública y Ministerio de Inclusión Económica y Social como entidades ejecutoras, el monto total se estimó en \$24.760.682,06 (VEINTE Y CUATRO MILLONES SETECIENTOS SESENTA MIL SEISCIENTOS OCHENTA Y DOS 06/100 Dólares de los Estados Unidos de América) alrededor de ocho millones más que su antecesora.

Es importante destacar que el plan familia sentaba sus bases en "la familia nuclear heterosexual y apela a un modelo familiar "positivo", a la abstinencia, e impulsa una educación enfocada en el arte de amar como solución a los altos índices de embarazo en adolescentes y situaciones de violenencia" (Zaragocin et al., 2018, p.113). De esta forma se argumentaba que la responsabilidad de la educación en materia de SSR recaía principalmente en la familia por tanto era importante fortalecer las figuras familiares tradicionales conformadas por un padre y una madre. Dentro del análisis situacional del cual parte el Plan Familia se indetifica una fuerte crítica a su antesesora en ENIPLA al mencionar que "Ios niños, niñas y adolescentes acceden a mucha información incompleta sobre aspectos de la sexualidad (especialmente relacionada con sus cuerpos y aspectos muy limitados del erotismo), lo cual no aporta a su enriquecimiento como personas porque con frecuencia se presenta una visión distorsionada de su dignidad"(Presidencia de la República del Ecuador, 2015, p.14). De igual manera se afirmó que la cultura inmediatista se centraba en mensajes con poco contenido en valores en donde se enfatizaba el hedonismo del "sexo seguro", la crítica también recayó en la promoción, a juicio de la Presidencia de la República, indiscriminada de anticonceptivos y preservativos a cualquier edad. Para Cifuentes (2016) este cambio no fue bien acogido por sectores progresista que manifestaron su fuerte rechazo a la nueva estrategia.

El objetivo del plan familia se centró en "prevenir el embarazo adolescente, fortaleciendo el rol protagónico de la familia y empoderando a los y las adolescentes de ma- 
nera que puedan tomar decisiones libres, informadas y responsables" (Presidencia de la República del Ecuador, 2015). Para cumplir con el objetivo se plantearon varias estrategias, como el fortalecimiento de capacidades del Ministerio de Salud Pública, a través de la capacitación a profesionales sobre afectivididad y sexualidad en donde se transversalice el enfoque familiar. Para el 2017, Lenin Moreno asumía la presidencia de la República y eliminó la estrategia intersectorial de su antecesor, esto de primera mano fue acogido con beneplácito y se lo tildó como "un triunfo para movimientos feministas que habían demostrado su rechazo a las políticas instauradas por Correa. Sin embargo, desde que Moreno eliminó el Plan Familia el país no cuenta con una política sexual ni con programas que fomenten los derechos sexuales y reproductivos" (Yépez Naranjo, 2021, p. 4).

A pesar del trabajo realizado desde la sociedad civil, tanto en la planificación de la ENIPLA como para instar la eliminación del Plan Familia, el análisis del aborto como un problema de salud pública fue abordado solamente en la ENIPLA en donde se estimó que "en algo más de una década se incrementó de once mil abortos a veinte y cinco mil, es decir más del doble" (Ministerio Coordinador de Desarrollo Social, 2011, p.18) cifras que ubicaban a Ecuador como el primer país con más abortos en condiciones de riesgo en toda América Latina. En el Plan Familia no se abordó la temática y aún menos se la consideró como un problema de orden público.

En este sentido, la Organización Mundial de la Salud (OMS) define como salud sexual el "estado de bienestar físico, mental y social en relación con la sexualidad, y no solamente la ausencia de enfermedad, disfunción o malestar" (Organización Mundial de la Salud, 2018, p.3) por tanto, es importante el abordaje de este concepto desde un enfoque de respeto a la decisión en libertad sin que de por medio exista coacción discri- minación o violencia. Para el pleno reconocimiento de los derechos a la salud sexual es indispensable, según la OMS, el reconocimiento de otros derechos humanos tanto en el ámbito internacional como regional o por los sistemas de protección de derechos humanos, estos incluyen entre otros, el derecho a la igualdad y no discriminación, el derecho a no ser sometidos a tratos crueles inhumanos o degradantes, así como también el derecho a decidir el número de hijos o hijas y el intervalo entre los tiempos de gestación, y el derecho a la información y educación.

Esta misma organización define el aborto inseguro como "un procedimiento para finalizar un embarazo no deseado realizado por personas que carecen de la capacidad necesaria o que se lleva a cabo en un entorno donde se carece de un estándar médico mínimo, o ambos" (Organización Mundial de la Salud, 2012).

Se estima que en America Latina se realizan entre 3.7 y 4.2 millones de abortos en condicion de inseguridad que terminan con el fallecimiento de la mujer (MSP el al.,2017). Ecuador engrosa las cifras de abortos clandestinos, pues se estima que es el país en donde con mayor frecuencia se realiza esta práctica en Latinoamérica estimándose alrededor de 95.000 mujeres que anualmente interrumpen sus embarazos (Guerra Rodríguez, 2018). En Ecuador el aborto es considerado un delito, así el Código Orgánico Integral Penal (COIP) establece que:

"La persona que haga abortar a una mujer que ha consentido en ello, será sancionada con pena privativa de libertad de uno a tres años. La mujer que cause su aborto o permita que otro se lo cause, será sancionada con pena privativa de libertad de seis meses a dos años" (COIP, 2014, art. 149).

Por su parte el articulo 150 del aborto no punible señala que:

"El aborto practicado por un médico u otro profesional de la sa- 
lud capacitado, que cuente con el consentimiento de la mujer o de su cónyuge, pareja, familiares íntimos o su representante legal, cuando ella no se encuentre en posibilidad de prestarlo, no será punible en los siguientes casos:

1. Si se ha practicado para evitar un peligro para la vida o salud de la mujer embarazada y si este peligro no puede ser evitado por otros medios.

2. Si el embarazo es consecuencia de una violación en una mujer que padezca de discapacidad mental" (COIP, 2014).

Bajo esta realidad jurídica se incrementa las prácticas persecutorias a las mujeres, es así que para el 2015 se presentaron 92 procesos penales por aborto consentido, en el 2016 se presentaron 43 caso y a junio de 2017, 62 casos (Zaragocin et al., 2018). Esta realidad que atraviesan las mujeres ecuatorianas se recrudece por factores transversales como la violencia y el encarecimiento de la vida. La penalización del aborto para Guerra Rodríguez (2016) tiene un impacto diferenciado en adolescentes pues estas sufren restricción en su autodeterminación desde una mirada adultocéntrica que determina la incapacidad de decidir sobre sus cuerpos.

Debido a esta problemática en América Latina y el Caribe y gracias a grupos de mujeres y colectivos feministas, el tema del aborto se reabre en la región y la posibilidad de despenalizarlo se plantea en la mesa de discusión política y legislativa.

Las diferentes posturas, antagónicas entre si, incidieron la toma de decisiones, es así que en el años 2013, un año antes del cambio de plan intersectorial por el plan familia, la postura conservadora del entonces mandatario del país se visibiliza de forma muy particular al amenazar con dimitir de su cargo y sancionando a las asambleístas de su partido por haber apoyado la propuesta de debatir de reforma del Código Orgánico
Integral Penal (COIP) que pretendía despenalizar el aborto por violación.

Con un gobierno nuevo en el 2017 se presenta un proyecto de ley contra violencia de las mujeres, lo que no es bien recibido por ciertos grupos conservadores que emprenden una serie de movilizaciones para luchar en contra de la ideología de genero y defender el concepto de familia heteronormada (Zaragocin et al., 2018).

La nueva iniciativa de los colectivos sociales de mujeres y feministas por despenalizar el aborto por causa de violación se ve estancada nuevamente en el 2019, con 65 votos a favor de la propuesta, 59 en contra y seis abstenciones, en el debate de las reformas al COIP.

Sin embargo, el 28 de abril de 2021 el Pleno de la Corte Constitucional del Ecuador emite la sentencia No. 34-19-IN/21 Y ACUMULADOS en donde analiza la constitucionalidad de los artículos 149 y 150 del COIP respecto a la penalización del aborto consentido en caso de mujeres víctimas de violación.

En esta sentencia se analiza la proporcionalidad de la sanción penal hacia mujeres que han sido víctimas de violación y han interrumpido su embarazo, de igual manera la sentencia analiza la protección del no nato frente al derecho a la integridad de niñas adolescentes y mujeres vícitmas de violación.

Es así que la Corte Constitucional resolvío "Declarar la inconstitucionalidad por el fondo del artículo 150 numeral 2 del Código Orgánico Integral Penal en la frase "en una mujer que padezca de discapacidad mental" (C.C. IN y acumulados sentencia No. 34-19-IN/21).

\section{Resultado}

Una vez analizados los objetivos de las dos políticas públicas, antagónicas entre si, resulta complejo analizar los resultados de las ENIPLA puesto que su interrupción en el 2014 no permitió alcanzar con los objetivos 
planteados. Para Ana Cristina Vera, experta en Derechos Sexuales y Reproductivos "el tema de reducir el embarazo adolescente no es a corto plazo. Eso no es posible. Reducir el embarazo adolescente pasa por muchos factores y no es algo que se pueda hacer en dos años" (Plan V, 2015). De igual manera manifiesta Rodríguez Yánez (2019) en sentido de no poder analizar los resultados pues no existe información consistente y mucha información ha desaparecido.

Para el año 2012 la tasa de nacidos vivos entre mujeres de 15 a 19 años era de 45.607 lo que representa el $19.39 \%$ de la población (Presidencia de la República del Ecuador, 2015). Por ello dentro del plan familia una de sus metas era que al 2017 se podría estabilizar el embarazo adolescente en torno al $19 \%$, sin embargo según la Tasa Específica de Fecundidad en Adolescentes (TEFA) para el 2017 por cada 1.000 mujeres de entre 10 y 19 años nacieron en promedio 3,6 hijos, cuando se desagrega por edad los resultados en el caso de niñas y adolescentes 10 a 14 años la TEFA fue de 2,84, lo que equivale a 2.298 nacidos vivos, mientras que la TEFA para adolescentes de entre 15 y 19 años fue de 70,9 , ascendiendo a un total de 54.715 nacidos vivos (Fondo de Población de las Naciones Unidas, 2020).

\section{Conclusiones}

En Ecuador, las políticas públicas en materia de salud sexual y reproductiva se han visto claramente marcadas por los discursos moralistas del quienes ostentan el poder, por tanto, no existe consistencia en la ejecución de las mismas. Claro ejemplo la finalización temprana de la ENIPLA y la posterior eliminación del Plan Familia.

Al no haber culminado ninguna de las estrategias, resulta complejo evaluarlas. Sin embargo, la estadística disponible refleja que el embarazo en adolescentes sigue siendo un problema de salud pública sin resolver.

Es importante también destacar que el índice de embarazo en adolescentes refleja el grado de violencia que sufren las mujeres en Ecuador. De igual forma se ha podido evidenciar el trabajo de colectivos sociales en las propuestas realizadas durante los mandatos analizados.

La violencia sexual que sufren niñas, adolescentes y mujeres en Ecuador, sigue siendo una deuda pendiente para el Estado, la reparación integral es un parámetro que con la última sentencia de la Corte Constitucional busca asidero en el marco normativo que está pendiente de construcción.

\section{Bibliografía}

Centro de Estudios de Población y Desarrollo Social (CEPAR). (2005). Encuesta demográfica y de salud materna e infantil.

Fondo de Población de las Naciones Unidas. (2020). Consecuencias socioeconómicas del embarazo en adolescentes en Ecuador. Implementación de la metodología para estimar el impacto socioeconómico del embarazo y la maternidad adolescentes en países de América Latina y el Caribe Milena 1.0. https://www.salud.gob.ec/wp-content/ uploads/2020/12/consecuencias_socioeconomicas_del_embarazo_adolescente_en_ecuador_1. pdf

Guardéras, P., \& Carófilis, C. (2020). Contrasentidos en las políticas contra la violencia de género y a los derechos sexuales y reproductivos en Ecuador. https://www.jstor.org/stable/pdf/j.ctv1gm02bg.10. pdf

Guerra Rodríguez, E. (2018). Implicaciones de la criminalización del aborto en Ecuador. FORO. Revista de Derecho, 117-134. https://doi. org/10.32719/26312484.2018.29.6

Habla serio, sexualidad sin misterios | La Iniciativa de Comunicación. (n.d.). Retrieved May 8, 2021, from https://www.comminit.com/la/content/habla-serio-sexualidad-sin-misterios

Ministerio Coordinador de Desarrollo Social. (2011). Estrategia Intersectorial de Prevención del Embarazo Adolescente y Planificación Familiar. In Estrategia Intersectorial de Prevención del Embarazo Adolescente y Planificación Familiar (Vol. 1). http:// www.todaunavida.gob.ec/wp-content/uploads/ downloads/2015/04/Proyecto_enipla.pdf

Ministerio de Inclusión Económica y Social. (2010). Dirección de la Juventud. In Ministerio De Inclusion Economica Y Social (Issue 593). 
Ministerio de Salud Pública;Senplades;UNFPA;SENDAS. (2017). Costos de Salud Sexual y Reproductiva en Ecuador. http://sendas.org.ec/documentos/ informe002.pdf

Organización Mundial de la Salud. (2012). Aborto sin riesgos: guia tenica y de politica para sistemas de salud. Organizacion Mundial de La Salud, 1(1), 123. www.who.int/reproductivehealth

Organización Mundial de la Salud. (2018). La salud sexual y su relación con la salud reproductiva: un enfoque operativo. http://apps.who.int/iris/bitstream/handle/10665/274656/9789243512884-spa. pdf?ua $=1$

Paz, D. F. (2018). Políticas de educación en sexualidad en el período 1998-2017. Sus sujetos y contextos en el caso ecuatoriano. RELAPAE, 8, 26-38.

Plan V. (2015). Plan Familia Ecuador o un retroceso de cien años | Plan V. Plan V. https://www.planv. com.ec/historias/testimonios/plan-familia-ecuador-o-un-retroceso-cien-anos

Presidencia de la República del Ecuador. (2015). "Plan Nacional De Fortalecimiento De La Familia."
Rodríguez, M. E. (2016). ¿Qué conocemos del derecho a la salud? Propuesta de marco conceptual en perspectiva crítica para Venezuela. https:// repositorio.uasb.edu.ec/bitstream/10644/5934/1/ SM210-Rodriguez-Que conocemos.pdf

Yépez, S. (2021). Vista de El "ejército de Dios" se moviliza en contra del mal: politización religiosa en contra del género y los feminismos en Ecuador. 61, 113-133. https://debatefeminista.cieg.unam. mx/df_ojs/index.php/debate_feminista/article/ view/2234/2023

Zaragocin, S., Cevallos, R., Falanga, G., Arrazola, I., Ruales, G., Vera, V., Yepez, A., La Criminalización Del Aborto En El Ecuador, M., \& Revista, L. (2018). Mapeando la criminalización del aborto en el Dossier Sobre el Aborto en Latinoamérica Mapping the criminalization of abortion in Ecuador Mapejant la criminalització de l'avortament a l'Equador Sofía Zaragocin, María Rosa Cevallos, Guglielmina Falanga, Iñigo Arrazola, Gabriela Ruales, Verónica Vera, Amanda Yepez Observatori De Bioètica I Dret De La Universitat De Barcelona. Rev Bio y Der, 43, 109-125. www.bioeticayderecho.ub.edu

\section{CITAR ESTE ARTICULO:}



CREATIVE COMMONS RECONOCIMIENTO-NOCOMERCIAL-COMPARTIRIGUAL 4.0.

Gamboa Vargas, S. L., \& Guerrero Salgado, E. E. (2021). Estrategias de políticas públicas de salud sexual y reproductiva. Despenalización del aborto en Ecuador. RECIAMUC, 5(4), 37-47. https://doi.org/10.47464/reciamuc/5.(4). noviembre.2021.37-47 